\title{
Growth rate, metabolic parameters and carcass quality in turkeys fed diets with different inclusion levels and sources of supplemental copper
}

\author{
K. Otowski', K. Ognik ${ }^{2}$ and K. Kozłowski ${ }^{1,3}$ \\ ${ }^{1}$ University of Warmia and Mazury, Department of Poultry Science, Oczapowskiego 5, 10-719 Olsztyn, Poland \\ ${ }^{2}$ University of Life Sciences in Lublin, Faculty of Biology, Animal Sciences and Bioeconomy, \\ Department of Biochemistry and Toxicology, Akademicka 13, 20-950 Lublin, Poland
}

KEY WORDS: copper, growth performance, meat quality, nanoparticles, turkeys

Received: 28 January 2019

Revised: $\quad 20$ July 2019

Accepted: 16 September 2019

${ }^{3}$ Corresponding author:

e-mail: kristof@uwm.edu.pl

\begin{abstract}
The aim of this experiment was to determine whether the inclusion levels of supplemental copper $(\mathrm{Cu})$ in turkey diets can be decreased without compromising important metabolic functions, growth parameters and carcass quality, and whether the above goals can be achieved with the involvement of Cu nanoparticles. The experiment was carried out on 648 one-day-old Hybrid Converter turkeys divided into 6 groups, with 6 replicates per group (18 birds per replicate). The experiment had a two-factorial design, with $\mathrm{Cu}$ sulphate (Cu-SUL) and $\mathrm{Cu}$ nanoparticles (CU-NP) as 2 dietary sources of $\mathrm{Cu}$, and 3 dietary inclusion levels of $\mathrm{Cu}(2,10$ and $20 \mathrm{mg} / \mathrm{kg})$. It was demonstrated that the replacement of Cu-SUL with Cu-NP and a decrease in a dose of supplemental Cu from 20 to $10 \mathrm{mg} / \mathrm{kg}$ or even $2 \mathrm{mg} / \mathrm{kg}$ of the diet did not affect the growth parameters or the carcass quality of turkeys. The few effects exerted by the substitution of Cu-SUL with Cu-NP included an increase in haemoglobin levels and an improvement in the antioxidant status of fresh breast meat. However, fresh meat was characterised by optimal redox parameters when the dietary dose of Cu was decreased to $10 \mathrm{mg} / \mathrm{kg}$. The results of the present study cannot be generalised, but they significantly expand the knowledge about Cu-NP as an efficient source of $\mathrm{Cu}$ for turkeys.
\end{abstract}

\section{Introduction}

In a modern agricultural practice, poultry diets are routinely supplemented with micronutrients, including copper $(\mathrm{Cu})$, because the $\mathrm{Cu}$ content of basic feed components does not fully meet the nutrient requirements of fast-growing birds. According to the latest recommendations, the diets of broiler turkeys should contain up to $30 \mathrm{mg} \mathrm{Cu} / \mathrm{kg}$ (Hybrid Turkeys, 2016), which is significantly more than the previously recommended $\mathrm{Cu}$ dose of $8 \mathrm{mg} / \mathrm{kg}$ (NRC, 1994) as well as the rates recommended by the European Commission's Scientific Committee for Animal Nutrition (European Commission, 2003). According to European Commission (2003), the dietary inclusion levels of available $\mathrm{Cu}$ from organic sources can be lower (up to $20 \mathrm{mg} \mathrm{Cu} / \mathrm{kg}$ of feed), and the total $\mathrm{Cu}$ content in animal diets should not exceed $35 \mathrm{mg} / \mathrm{kg}$. The European Food Safety Authority (EFSA FEEDAP Panel, 2016) has recently published the newly proposed maximum content (NPMC) of $\mathrm{Cu}$ in complete feeds for target animals. The NPMC for poultry, including turkeys for fattening, was set at $25 \mathrm{mg} / \mathrm{kg}$. 
According to some postulates, the recommended doses of supplemental $\mathrm{Cu}$ should be decreased because $\mathrm{Cu}$ excreted with animal faeces increases the $\mathrm{Cu}$ load in the environment. Research has demonstrated that the excretion of $\mathrm{Cu}$ and other minerals increased linearly with an increase in their dietary intake (Bao et al., 2007). In laying hens, the $\mathrm{Cu}$ content per $\mathrm{kg}$ of excreta dry matter (DM) increased from 25.3 to $397 \mathrm{mg} / \mathrm{kg}$ when the basal diet was supplemented with $\mathrm{CuSO}_{4}(\mathrm{Cu}-\mathrm{SUL})$ at 0 and $240 \mathrm{mg} / \mathrm{kg}$ (Skřivan et al., 2006). In many experiments it was demonstrated that organic trace elements, including amino acid chelates, increase nutrient bioavailability (Leeson and Caston, 2008; Jegede et al., 2011) and decrease faecal nutrient losses (Mikulski et al., 2009). Amino acid chelates are absorbed from the intestines at a significantly higher rate than soluble inorganic metal salts, but their supplementation is often difficult and not economically viable (Andersen, 2004). Copper nanoparticles (Cu-NP) are a new and alternative source of dietary $\mathrm{Cu}$ with potentially high availability (Gonzales-Eguia etal., 2009; Ogniketal., 2016;2018; Kozłowski et al., 2018; Jankowski et al., 2019). The metabolic rate and development of broiler embryos were improved when $\mathrm{Cu}-\mathrm{NP}$ were injected in ovo or included in hen diets (Pineda et al., 2013). Diets supplemented with more available nanoparticles (44.0 vs $34.2 \%$ for $\mathrm{CuSO}_{4}$ ) improved growth performance in piglets (Gonzales-Eguia et al., 2009), but similar experiments are rarely conducted on growing poultry. In our preliminary study on young turkeys, a decrease in the dose of supplemental $\mathrm{Cu}$, both $\mathrm{Cu}-\mathrm{SUL}$ and $\mathrm{Cu}-\mathrm{NP}$, from 20 to $2 \mathrm{mg} / \mathrm{kg}$ of the diet did not compromise growth parameters. Copper in a dose of $20 \mathrm{mg} / \mathrm{kg}$ induced oxidation reactions and was less effective in conferring antioxidant protection than $\mathrm{Cu}$ in a dose of $2 \mathrm{mg} / \mathrm{kg}$. Dietary supplementation with $\mathrm{Cu}-\mathrm{NP}$ also exerted a more beneficial influence on the carbohydrate metabolism and antioxidant status of young turkeys than supplementation with conventional Cu-SUL (Kozłowski et al., 2018). According to Sawosz et al. (2018), $\mathrm{Cu}$ inclusion levels in poultry diets can be decreased and environmental $\mathrm{Cu}$ loads can be minimised by replacing $\mathrm{Cu}-\mathrm{SUL}$ with $\mathrm{Cu}-\mathrm{NP}$ without compromising growth parameters.

The aim of this study was to determine whether the inclusion levels of supplemental $\mathrm{Cu}$ in turkey diets can be decreased without compromising important metabolic functions, growth parameters and carcass quality, and whether the above goals can be achieved with the involvement of $\mathrm{Cu}-\mathrm{NP}$.

\section{Material and methods}

\section{Birds, management and diet}

The experiment and the slaughter protocol were approved by the local Ethical Committee for Experiments on Animals in Olsztyn (permission No. 30/2015; 2015.04.29). In total, 648 one-dayold Hybrid Converter female turkeys were placed in 36 pens and kept according to the breeder's recommendations that were adjusted for the birds' age. Turkeys were divided into 6 groups, with 6 replicates per group (18 birds per replicate). The experiment had a two-factorial design, with copper sulphate $(\mathrm{Cu}-\mathrm{SUL})$ and $\mathrm{Cu}$ nanoparticles $(\mathrm{Cu}-\mathrm{NP})$ as 2 dietary sources of $\mathrm{Cu}$, and 3 dietary inclusion levels of $\mathrm{Cu}(2,10$ and $20 \mathrm{mg} / \mathrm{kg})$. Turkeys had free access to water and feed that was prepared locally by the 'Agrocentrum' Feed Mill Ltd. Crumbled (1-28 days of age) and pelleted (next feeding stage) experimental diets (Table 1) were supplemented

Table 1. The composition and nutrient content of experimental diets, $\%$ as-fed basis

\begin{tabular}{|c|c|c|c|}
\hline \multirow{2}{*}{ Indices } & \multicolumn{3}{|c|}{ Feeding period, days } \\
\hline & $1-42$ & $43-70$ & $71-98$ \\
\hline \multicolumn{4}{|l|}{ Ingredients } \\
\hline wheat & 43.11 & 46.20 & 61.66 \\
\hline soybean meal & 38.97 & 30.46 & 15.95 \\
\hline faba bean & 10.00 & 10.00 & 10.00 \\
\hline rapeseeds & - & 5.00 & 6.00 \\
\hline soybean oil & 2.80 & 3.86 & 3.54 \\
\hline sodium sulphate & 0.15 & 0.15 & 0.15 \\
\hline salt & 0.20 & 0.16 & 0.17 \\
\hline limestone & 1.60 & 1.57 & 0.85 \\
\hline monocalcium phosphate & 1.75 & 1.32 & 0.67 \\
\hline L-methionine, $99 \%$ & 0.37 & 0.26 & 0.20 \\
\hline DL-lysine $\mathrm{HCl}, 75 \%$ & 0.44 & 0.40 & 0.37 \\
\hline L-threonine, 99\% & 0.12 & 0.12 & 0.05 \\
\hline mineral-vitamin premix ${ }^{1}$ & 0.50 & 0.50 & 0.40 \\
\hline \multicolumn{4}{|l|}{ Calculated nutrient density } \\
\hline crude protein & 26.50 & 23.00 & 18.50 \\
\hline crude fibre & 3.40 & 3.98 & 3.57 \\
\hline crude fat & 4.23 & 7.16 & 7.37 \\
\hline $\mathrm{AME}^{2}, \mathrm{kcal} / \mathrm{kg}$ & 2750 & 2950 & 3100 \\
\hline arginine & 1.76 & 1.52 & 1.18 \\
\hline lysine & 1.74 & 1.50 & 1.17 \\
\hline methionine & 0.71 & 0.57 & 0.45 \\
\hline Met + Cys & 1.13 & 0.95 & 0.78 \\
\hline threonine & 1.05 & 0.93 & 0.68 \\
\hline tryptophan & 0.32 & 0.29 & 0.22 \\
\hline $\mathrm{Ca}$ & 1.15 & 1.05 & 0.65 \\
\hline$P$ & 0.55 & 0.45 & 0.30 \\
\hline $\mathrm{Na}$ & 0.15 & 0.13 & 0.13 \\
\hline
\end{tabular}

${ }^{1}$ per $\mathrm{kg}$ of diet: IU: vit. A 24999.75, vit. D 35000, vit. E 100; mg: tocopherol 91 , vit. $K 4$, vit. $B_{1} 5$, vit. $B_{2} 15$, vit. $B_{6} 6$, vit. $B_{12} 0.04$, niacin 100 , pantothenic acid 30 , folic acid 4 , choline chloride 700 , calcium D-pantothenate 32.665 , biotin 0.35 , total Se 0.3 , total Fe 60 , total Mn 100, total Zn 100, J 1.5; g: Ca 1.0435; ${ }^{2} \mathrm{AME}-$ Apparent Metabolizable Energy 
with vitamin-mineral premixes containing different amounts and sources of $\mathrm{Cu}$. The Cu-SUL diet contained conventional copper sulphate, and the $\mathrm{Cu}-\mathrm{NP}$ diet contained $\mathrm{Cu}$ nanoparticles $(25 \mathrm{~nm}$ in size) powder with $99.8 \%$ purity (purchased from Sky Spring Nanomaterials Inc., Houston, TX, USA). The tested $\mathrm{Cu}$ supplements were added to the vitamin-mineral premix using a carbohydrate carrier (glucose).

\section{Growth trial and sample collection}

Body weights (BW), body weight gains (BWG) and feed intake were recorded and calculated on a pen basis. Daily feed intake (DFI) per bird was calculated based on total feed consumption per pen for the entire experimental period and on selected days of the experiment. The feed conversion ratio (FCR; $\mathrm{kg}$ of feed $/ \mathrm{kg}$ of BWG) was calculated per pen based on BWG and feed intake. Mortality rates, including the cause of death, were recorded daily, and the BW of dead birds were used to adjust the average BWG, average DFI and FCR.

After 14 weeks of feeding, seven turkeys from each group (with an average BW of the group) were slaughtered at 98 day of age at the Department's slaughterhouse, $8 \mathrm{~h}$ after feed withdrawal. Before slaughter, blood was collected from the wing vein into test tubes with an anticoagulant (heparin). Blood samples were centrifuged at $3000 \mathrm{~g}$ for $10 \mathrm{~min}$, and plasma was collected for further analysis. Subsamples of the pectoralis major muscle were used to determine meat colour upon deboning ( $24 \mathrm{~h}$ post mortem). The remaining portion of breast meat was vacuum-packaged, frozen at $-20^{\circ} \mathrm{C}$, and stored for further analysis.

\section{Laboratory analyses}

The $\mathrm{Cu}$ contents in feed and tissue (blood, liver and meat) samples were determined by inductively coupled plasma optical emission spectrometry (ICP-OES, Varian Inc., Palo Alto, CA, USA). The contents of zinc $(\mathrm{Zn})$, calcium $(\mathrm{Ca})$, phosphorus (P) and magnesium $(\mathrm{Mg})$ were determined by flame atomic absorption spectrometry (FAAS, Varian Inc., Palo Alto, CA, USA). Haemoglobin $(\mathrm{Hb})$ and haematocrit $(\mathrm{Ht})$ levels were measured using an automatic haematology analyser (Abacus Junior Vet, Diatron, Budapest, Hungary). The concentrations of glucose (GLU), triacylglycerols (TAG), total cholesterol (TC), uric acid (UA), urea (UREA) and total protein (TP), and the activity of alanine aminotransferase (ALT), aspartate aminotransferase (AST), alkaline phosphatase (ALP) and gamma-glutamyl transferase (GGT) were measured in the blood plasma of turkeys using an automatic biochemical analyser (Plasma Diagnostic Instruments Horiba, Kyoto, Japan).

The yields of whole carcasses, breast muscles and thigh muscles were determined relative to live BW. The colour of breast muscles was determined $24 \mathrm{~h}$ post mortem by the optical reflection method in the CIELAB system, where the values of $\mathrm{L}^{*}$ (lightness, lower values indicate a darker colour), $\mathrm{a}^{*}$ (redness, higher positive values indicate a higher contribution of redness) and $b^{*}$ (yellowness, higher positive values indicate a higher contribution of yellowness) were measured with the MiniScan XE Plus portable spectrophotometer (Hunter Associates Laboratory, Inc., Reston, VA, USA). The average of two readouts from the cross-section of each right breast muscle free from colour defects, bruising and haemorrhages was recorded. The redox status of turkey breast meat was assessed using the methods described by Ognik and Wertelecki (2012), and the following indicators were determined: antioxidant indicators: superoxide dismutase (SOD) and catalase (CAT) activity, total glutathione (GSH + GSSG) content and oxidant indicator: malondialdehyde (MDA) content.

\section{Statistical analysis}

A single pen $(n=6)$ was regarded as a replicate experimental unit in a statistical analysis of performance parameters. Individual birds were regarded as experimental units in analyses of the biochemical and antioxidant parameters of tissues. The biochemical and antioxidant parameters of the blood plasma were analysed in 36 birds representing 6 replications from each of the 6 experimental treatments. Two-way ANOVA was performed to determine the effects of different inclusion levels $(2,10$ and $20 \mathrm{mg} / \mathrm{kg}$ ) and sources of supplemental $\mathrm{Cu}(\mathrm{Cu}-\mathrm{SUL}$ or $\mathrm{Cu}-\mathrm{NP})$, and to determine the interactions between both factors (inclusion level $\times$ source; IL $\times S$ ). In significant IL $\times$ S interactions, the significance of differences between the mean values of the analysed parameters in groups was estimated by Tukey's multiple-range test. Treatment effects were considered to be significant at $P<0.05$. The results were processed in the Statistica PL ver. 13.1 (StatSoft Corp., Kraków, Poland) application.

\section{Results}

The $\mathrm{Cu}$ content in all experimental diets approximated the values assumed in the experimental design (Table 2). The observed minor differences between groups could have resulted from different 
Table 2. The content of $\mathrm{Cu}$ and selected minerals in turkey diets

\begin{tabular}{|c|c|c|c|}
\hline \multirow{2}{*}{ Indices } & \multicolumn{3}{|c|}{ Feeding period, days } \\
\hline & $1-42$ & $43-70$ & $71-98$ \\
\hline \multicolumn{4}{|c|}{ Cu content of experimental diets ${ }^{1}, \mathrm{mg} / \mathrm{kg}$} \\
\hline Cu-SUL 20 & 31.2 & 29.1 & 30.7 \\
\hline $\mathrm{Cu}-\mathrm{NP}_{20}$ & 28.4 & 27.2 & 26.9 \\
\hline $\mathrm{Cu}-S U L_{10}$ & 21.1 & 17.9 & 18.8 \\
\hline Cu-NP 10 & 20.4 & 18.3 & 17.6 \\
\hline $\mathrm{Cu}_{\mathrm{SUL}}$ & 14.9 & 12.6 & 12.9 \\
\hline Cu-NP${ }_{2}$ & 13.7 & 13.4 & 12.5 \\
\hline \multicolumn{4}{|c|}{ Content of selected minerals in the diet, $\mathrm{mg} / \mathrm{kg}$} \\
\hline $\mathrm{Ca}$ & 12.8 & 11.5 & 7.10 \\
\hline$P$ & 8.70 & 7.90 & 5.20 \\
\hline $\mathrm{Zn}$ & 172 & 140 & 148 \\
\hline $\mathrm{Fe}$ & 258 & 229 & 221 \\
\hline
\end{tabular}

${ }^{1}$ diets supplemented with 2, 10 and $20 \mathrm{mg}$ of copper sulphate (Cu-SUL $\left.{ }_{2}, \mathrm{Cu}-\mathrm{SUL}_{10}, \mathrm{Cu}-\mathrm{SUL}_{20}\right)$ or copper nanoparticles (Cu-NP $\mathrm{Cu}-\mathrm{NP}_{10}, \mathrm{Cu}-\mathrm{NP}_{20}$ )

$\mathrm{Cu}$ concentrations in the feed ingredients used for formulating diets in successive stages of the feeding trial. The difference between the total $\mathrm{Cu}$ content of diets and supplemental $\mathrm{Cu}$ doses indicated that the major feed ingredients supplied approximately $11 \mathrm{mg} / \mathrm{kg} \mathrm{Cu}$ in total. The basal diet supplemented with different doses of $\mathrm{Cu}$ supplied identical amounts of $\mathrm{Ca}, \mathrm{P}, \mathrm{Zn}$ and $\mathrm{Fe}$ to the experimental diets, and the concentrations of these nutrients decreased as the birds grew older.

The applied dietary treatments had no influence on $\mathrm{Ca}$ and $\mathrm{Mg}$ concentrations in the blood plasma (Table 3). Regardless of the $\mathrm{Cu}$ source, plasma $\mathrm{P}$ concentration was higher in the treatment with the lowest $\mathrm{Cu}$ supplementation $(2 \mathrm{mg} / \mathrm{kg})$ than in both treatments with higher supplementation levels $(10$ and $20 \mathrm{mg} / \mathrm{kg})$. An interaction between the inclusion level and the source of $\mathrm{Cu}$ was observed in plasma $\mathrm{Zn}$ concentrations $(P<0.001)$ which decreased in birds whose diets were supplemented with 20 and $10 \mathrm{mg}$ of $\mathrm{Cu}-\mathrm{NP} / \mathrm{kg}$ (vs Cu-SUL), whereas an opposite effect was noted in birds whose diets were supplemented with $2 \mathrm{mg}$ of $\mathrm{Cu}-\mathrm{NP} / \mathrm{kg}$. The above interaction was also observed in plasma $\mathrm{Cu}$ concentrations which were comparable in all four groups receiving 10 and $2 \mathrm{mg} / \mathrm{kg}$ of supplemental $\mathrm{Cu}$, whereas the highest and the lowest $\mathrm{Cu}$ plasma concentrations were noted in groups $\mathrm{Cu}-\mathrm{NP}_{20}$ and $\mathrm{Cu}-\mathrm{SUL}_{20}$, respectively $(P<0.05)$.

The values of $\mathrm{Ht}$ and the most of the analysed blood biochemical indicators, including uric acid levels and the activity of ALT, AST, GGT and ALP, were similar in all groups of birds whose diets were supplemented with different amounts of $\mathrm{Cu}-\mathrm{SUL}$
Table 3. The content of selected minerals in the blood plasma of turkeys

\begin{tabular}{|c|c|c|c|c|c|}
\hline \multirow[b]{2}{*}{ Indices } & \multicolumn{5}{|c|}{ Minerals } \\
\hline & $\begin{array}{l}\mathrm{Cu}, \\
\mu \mathrm{mol} / \mathrm{l}\end{array}$ & $\begin{array}{l}Z \mathrm{Zn}, \\
\mu \mathrm{mol} / \mathrm{I}\end{array}$ & $\begin{array}{l}\mathrm{Ca}, \\
\mathrm{mmol} / \mathrm{l}\end{array}$ & $\begin{array}{l}\mathrm{P}, \\
\mathrm{mmol} / \mathrm{l}\end{array}$ & $\begin{array}{l}\mathrm{Mg}, \\
\mathrm{mmol} / \mathrm{l}\end{array}$ \\
\hline \multicolumn{6}{|l|}{ Group ${ }^{1}$} \\
\hline Cu-SUL 20 & $2.81^{b}$ & $39.8^{\mathrm{a}}$ & 2.44 & 1.98 & 0.83 \\
\hline Cu-NP 20 & $4.62^{\mathrm{a}}$ & $36.3^{b}$ & 2.38 & 2.28 & 0.81 \\
\hline Cu-SUL $_{10}$ & $3.93^{\mathrm{ab}}$ & $38.9^{a}$ & 2.13 & 2.16 & 0.85 \\
\hline Cu-NP 10 & $3.96^{\mathrm{ab}}$ & $36.5^{b}$ & 2.33 & 2.28 & 0.94 \\
\hline $\mathrm{Cu}-\mathrm{SUL}_{2}$ & $3.70^{\mathrm{ab}}$ & $35.8^{b}$ & 2.19 & 2.62 & 0.93 \\
\hline $\mathrm{Cu}-\mathrm{NP}_{2}$ & $3.36^{\mathrm{ab}}$ & $39.0^{\mathrm{a}}$ & 2.08 & 2.70 & 0.86 \\
\hline \multicolumn{6}{|l|}{ Cu source } \\
\hline Cu-SUL & 3.48 & 38.1 & 2.25 & 2.26 & 0.87 \\
\hline Cu-NP & 3.98 & 37.3 & 2.26 & 2.42 & 0.87 \\
\hline \multicolumn{6}{|l|}{ Cu inclusion level, mg/kg } \\
\hline 20 & 3.72 & 38.0 & 2.41 & $2.13^{b}$ & 0.82 \\
\hline 10 & 3.94 & 37.7 & 2.23 & $2.22^{b}$ & 0.89 \\
\hline 2 & 3.53 & 37.4 & 2.14 & $2.66^{a}$ & 0.90 \\
\hline SEM & 0.179 & 0.380 & 0.054 & 0.067 & 0.019 \\
\hline \multicolumn{6}{|l|}{$P$-values } \\
\hline Cu source (S) & 0.147 & 0.186 & 0.916 & 0.163 & 0.990 \\
\hline Cu inclusion level (IL) & 0.610 & 0.748 & 0.128 & 0.001 & 0.160 \\
\hline $\mathrm{IL} \times \mathrm{S}$ interaction & 0.028 & $<0.001$ & 0.446 & 0.705 & 0.248 \\
\hline
\end{tabular}

1 diets supplemented with 20,10 and $2 \mathrm{mg}$ of copper sulphate (Cu-SUL $\left.{ }_{20}, \mathrm{Cu}-\mathrm{SUL}_{10}, \mathrm{Cu}-\mathrm{SUL}_{2}\right)$ or copper nanoparticles $\left(\mathrm{Cu}-\mathrm{NP}_{20}\right.$,

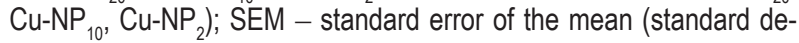
viation for all birds divided by the square root of the number of birds, $n=36$ ); $a, b$ - two-way ANOVA was applied, means within the same column with different superscript letters are significantly different across groups $(P \leq 0.05)$ in Tukey's test (calculated only if the $\mathrm{IL} \times \mathrm{S}$ interaction was significant)

and $\mathrm{Cu}-\mathrm{NP}$ (Table 4). Two-way ANOVA revealed that $\mathrm{Cu}-\mathrm{NP}$ supplementation significantly increased blood $\mathrm{Hb}$ concentrations relative to the $\mathrm{Cu}-\mathrm{SUL}$ treatment. Regardless of the source of supplemental $\mathrm{Cu}$, the lowest $\mathrm{Cu}$ level of $2 \mathrm{mg} / \mathrm{kg}$ significantly increased plasma $\mathrm{TC}$ concentrations relative to the two higher inclusion levels of $\mathrm{Cu}$.

After 16 weeks of the feeding trial during which turkey diets were supplemented with different amounts and sources of $\mathrm{Cu}$, none of the experimental factors affected BW (Table 5) or the carcass quality of turkeys (Table 6). Neither the source nor the inclusion level of supplemental $\mathrm{Cu}$ in the experimental diets affected mortality rates, BW, feed intake or the FCR in any of the three analysed rearing periods. The FCR values across treatments revealed marginally significant interactions between the source and inclusion level of $\mathrm{Cu}(\mathrm{P}=0.052)$ only during the entire experiment (days 1-98) because lower $\mathrm{Cu}-\mathrm{NP}$ doses induced a greater decrease in FCR values than the corresponding doses of Cu-SUL. The dressing 
Table 4. Haematological and biochemical parameters in the blood of turkeys ${ }^{1}$

\begin{tabular}{|c|c|c|c|c|c|c|c|c|c|c|c|c|}
\hline Indices & $\begin{array}{l}\mathrm{Ht}, \\
\mathrm{l} / \mathrm{I}\end{array}$ & $\begin{array}{l}\mathrm{Hb}, \\
\mathrm{g} / \mathrm{l}\end{array}$ & $\begin{array}{l}\text { GLU, } \\
\mathrm{mmol} / \mathrm{l}\end{array}$ & $\begin{array}{l}\text { TP, } \\
\text { g/l }\end{array}$ & $\begin{array}{l}\text { UREA, } \\
\mathrm{mmol} / \mathrm{l}\end{array}$ & $\begin{array}{l}\text { TAG, } \\
\mathrm{mmol} / \mathrm{l}\end{array}$ & $\begin{array}{l}\mathrm{TC}, \\
\mathrm{mmol} / \mathrm{l}\end{array}$ & $\begin{array}{l}\text { UA, } \\
\mu \mathrm{mol} / / \mathrm{l}\end{array}$ & $\begin{array}{l}\text { ALT, } \\
\mathrm{U} / \mathrm{I}\end{array}$ & $\begin{array}{l}\text { AST, } \\
\text { U/I }\end{array}$ & $\begin{array}{l}\text { GGT, } \\
\text { U/I }\end{array}$ & $\begin{array}{l}\text { ALP, } \\
\text { U/I }\end{array}$ \\
\hline \multicolumn{13}{|l|}{ Group $^{2}$} \\
\hline Cu-SUL $_{20}$ & 38.1 & 9.64 & 18.6 & 31.4 & 0.28 & 0.46 & 2.64 & 227 & 6.18 & 274 & 3.19 & 1422 \\
\hline Cu-NP 20 & 40.4 & 10.9 & 17.6 & 28.7 & 0.30 & 0.55 & 2.47 & 174 & 5.34 & 217 & 3.44 & 1399 \\
\hline Cu-SUL $_{10}$ & 38.5 & 10.3 & 16.6 & 31.2 & 0.34 & 0.49 & 2.40 & 184 & 6.63 & 208 & 2.66 & 1419 \\
\hline $\mathrm{Cu}-\mathrm{NP}_{10}$ & 38.0 & 10.5 & 17.5 & 33.5 & 0.38 & 0.54 & 2.39 & 219 & 5.33 & 237 & 4.09 & 1464 \\
\hline $\mathrm{Cu} \mathrm{SUL}_{2}$ & 37.8 & 10.1 & 18.2 & 34.2 & 0.42 & 0.51 & 2.84 & 216 & 4.29 & 307 & 2.89 & 1500 \\
\hline $\mathrm{Cu}-\mathrm{NP}_{2}$ & 38.5 & 10.6 & 18.7 & 30.9 & 0.32 & 0.69 & 2.77 & 218 & 5.93 & 245 & 2.84 & 1466 \\
\hline \multicolumn{13}{|l|}{ Cu source } \\
\hline Cu-SUL & 38.2 & $10.0^{b}$ & 17.8 & 32.3 & 0.35 & 0.48 & 2.62 & 209 & 5.70 & 263 & 2.91 & 1447 \\
\hline Cu-NP & 39.0 & $10.6^{a}$ & 17.9 & 31.0 & 0.33 & 0.59 & 2.55 & 204 & 5.23 & 233 & 3.45 & 1443 \\
\hline \multicolumn{13}{|l|}{ Cu inclusion level, mg/kg } \\
\hline 20 & 39.3 & 10.3 & 18.1 & 30.1 & 0.29 & 0.50 & $2.56^{b}$ & 200 & 5.76 & 246 & 3.31 & 1410 \\
\hline 10 & 38.3 & 10.4 & 17.1 & 32.4 & 0.36 & 0.52 & $2.40^{\mathrm{b}}$ & 202 & 5.98 & 223 & 3.38 & 1441 \\
\hline 2 & 38.2 & 10.3 & 18.4 & 32.5 & 0.37 & 0.60 & $2.80^{\mathrm{a}}$ & 217 & 5.11 & 276 & 2.86 & 1483 \\
\hline SEM & 0.309 & 0.135 & 0.299 & 0.659 & 0.022 & 0.030 & 0.051 & 10.42 & 0.267 & 10.63 & 0.253 & 15.00 \\
\hline \multicolumn{13}{|l|}{$P$-values } \\
\hline Cu source (S) & 0.181 & 0.023 & 0.861 & 0.343 & 0.733 & 0.076 & 0.409 & 0.802 & 0.748 & 0.142 & 0.300 & 0.883 \\
\hline Cu inclusion level (IL) & 0.265 & 0.898 & 0.149 & 0.225 & 0.288 & 0.375 & 0.004 & 0.764 & 0.367 & 0.105 & 0.678 & 0.151 \\
\hline $\mathrm{IL} \times \mathrm{S}$ interaction & 0.182 & 0.228 & 0.389 & 0.157 & 0.382 & 0.660 & 0.770 & 0.246 & 0.054 & 0.125 & 0.473 & 0.515 \\
\hline
\end{tabular}

${ }^{1}$ data represent the mean values of 6 birds per treatment; ${ }^{2}$ diets supplemented with 20,10 and $2 \mathrm{mg}$ of copper sulphate $\left(\mathrm{Cu}_{\mathrm{SUL}} \mathrm{SU}_{20}, \mathrm{Cu}_{\mathrm{SUL}}\right.$, Cu-SUL $\left.{ }_{2}\right)$ or copper nanoparticles $\left(\mathrm{Cu}_{-\mathrm{NP}_{2}}, \mathrm{Cu}-\mathrm{NP}_{10}, \mathrm{Cu}-\mathrm{NP}_{2}\right)$; SEM - standard error of the mean (standard deviation for all birds divided by the square root of the number of birds, $n=36$ ); Ht - haematocrit; Hb - haemoglobin; GLU - glucose; TP - total protein; ALB - albumin; TAG - triacylglycerols; TC - total cholesterol; UA - uric acid; ALT - alanine aminotransferase; AST - aspartate aminotransferase; GGT - gammaglutamyl transferase; ALP - alkaline phosphatase; a,b - two-way ANOVA was applied; - means within the same column with different superscript letters are significantly different across groups $(P \leq 0.05)$ in Tukey's test (calculated only if the $\mathrm{IL} \times \mathrm{S}$ interaction was significant)

Table 5. Growth performance of turkeys

\begin{tabular}{|c|c|c|c|c|c|c|c|c|c|c|c|c|c|}
\hline \multirow{2}{*}{ Indices } & \multicolumn{4}{|c|}{ Body weight, $\mathrm{kg}$} & \multicolumn{4}{|c|}{ Daily feed intake, g per bird } & \multicolumn{4}{|c|}{$\mathrm{FCR}, \mathrm{kg} / \mathrm{kg}$} & \multirow{2}{*}{$\begin{array}{l}\text { Mort., } \\
\%\end{array}$} \\
\hline & $\mathrm{MC}_{1}$ & $\mathrm{MC}_{42}$ & $\mathrm{MC}_{70}$ & $\mathrm{MC}_{98}$ & $1-42$ & $41-70$ & $71-98$ & $1-98$ & $1-42$ & $41-70$ & $71-98$ & $1-98$ & \\
\hline \multicolumn{14}{|l|}{ Group ${ }^{1}$} \\
\hline Cu-SUL 20 & 0.07 & 2.74 & 5.93 & 9.55 & 96.0 & 242 & 348 & 244 & 1.52 & 2.09 & 3.08 & 2.18 & 2.78 \\
\hline Cu-NP 20 & 0.07 & 2.79 & 5.97 & 9.55 & 98.5 & 246 & 353 & 257 & 1.52 & 2.17 & 3.20 & 2.21 & 1.85 \\
\hline Cu-SUL $_{10}$ & 0.07 & 2.69 & 5.97 & 9.51 & 97.4 & 249 & 354 & 245 & 1.56 & 2.11 & 3.20 & 2.24 & 0.00 \\
\hline Cu-NP 10 & 0.07 & 2.73 & 5.91 & 9.46 & 97.6 & 246 & 352 & 252 & 1.54 & 2.10 & 3.18 & 2.19 & 0.93 \\
\hline Cu-SUL ${ }_{2}$ & 0.07 & 2.75 & 5.89 & 9.43 & 98.9 & 243 & 365 & 258 & 1.55 & 2.16 & 3.36 & 2.25 & 0.93 \\
\hline Cu-NP ${ }_{2}$ & 0.07 & 2.76 & 5.97 & 9.56 & 99.2 & 243 & 351 & 251 & 1.55 & 2.09 & 3.13 & 2.17 & 0.93 \\
\hline \multicolumn{14}{|l|}{ Cu source } \\
\hline CU-SUL & 0.07 & 2.73 & 5.93 & 9.49 & 97.4 & 245 & 356 & 249 & 1.54 & 2.12 & 3.22 & 2.22 & 1.24 \\
\hline Cu-NP & 0.07 & 2.76 & 5.95 & 9.53 & 98.4 & 245 & 352 & 253 & 1.54 & 2.12 & 3.17 & 2.19 & 1.24 \\
\hline \multicolumn{14}{|l|}{ Cu inclusion level, mg/kg } \\
\hline 20 & 0.07 & 2.77 & 5.95 & 9.55 & 97.3 & 244 & 351 & 250 & 1.52 & 2.13 & 3.14 & 2.19 & 2.32 \\
\hline 10 & 0.07 & 2.71 & 5.94 & 9.49 & 97.5 & 247 & 353 & 249 & 1.55 & 2.10 & 3.19 & 2.22 & 0.46 \\
\hline 2 & 0.07 & 2.76 & 5.93 & 9.50 & 99.1 & 243 & 358 & 255 & 1.55 & 2.13 & 3.25 & 2.21 & 0.93 \\
\hline SEM & $<0.001$ & 0.014 & 0.023 & 0.043 & 0.429 & 2.165 & 2.866 & 2.140 & 0.006 & 0.018 & 0.037 & 0.009 & 0.449 \\
\hline \multicolumn{14}{|l|}{$P$-values } \\
\hline Cu source (S) & 0.599 & 0.228 & 0.662 & 0.733 & 0.252 & 0.953 & 0.491 & 0.369 & 0.477 & 0.980 & 0.528 & 0.074 & 0.866 \\
\hline Cu inclusion level (IL) & 0.133 & 0.246 & 0.944 & 0.828 & 0.184 & 0.729 & 0.603 & 0.511 & 0.149 & 0.779 & 0.509 & 0.529 & 0.272 \\
\hline $\mathrm{IL} \times \mathrm{S}$ interaction & 0.533 & 0.783 & 0.487 & 0.741 & 0.458 & 0.774 & 0.396 & 0.173 & 0.609 & 0.238 & 0.177 & 0.052 & 0.781 \\
\hline
\end{tabular}

${ }^{1}$ diets supplemented with 20,10 and $2 \mathrm{mg}$ of copper sulphate $\left(\mathrm{Cu}-\mathrm{SUL}_{20}, \mathrm{Cu}-\mathrm{SUL}{ }_{10}, \mathrm{Cu}-\mathrm{SUL}_{2}\right)$ or copper nanoparticles $\left(\mathrm{Cu}-\mathrm{NP}_{20}, \mathrm{Cu}_{\mathrm{NP}}{ }_{10}\right.$, $\mathrm{Cu}-\mathrm{NP}_{2}$ ); SEM - standard error of the mean (standard deviation for all birds divided by the square root of the number of birds, $\mathrm{n}=36$ ) 
Table 6. The results of turkey carcass analysis, relative weights of selected organs (body weight $=100 \%$ ) and breast meat colour

\begin{tabular}{|c|c|c|c|c|c|c|c|c|}
\hline \multirow{2}{*}{ Indices } & \multirow{2}{*}{$\begin{array}{l}\text { Dressing } \\
\text { percentage }\end{array}$} & \multirow{2}{*}{$\begin{array}{l}\text { Breast } \\
\text { muscle }\end{array}$} & \multirow{2}{*}{$\begin{array}{l}\text { Thigh } \\
\text { muscle }\end{array}$} & \multirow{2}{*}{$\begin{array}{l}\text { Drumstick } \\
\text { muscle }\end{array}$} & \multirow{2}{*}{$\begin{array}{l}\text { Abdominal } \\
\text { fat }\end{array}$} & \multicolumn{3}{|c|}{ Breast meat colour } \\
\hline & & & & & & $L$ & $a$ & $b$ \\
\hline \multicolumn{9}{|l|}{ Group ${ }^{1}$} \\
\hline $\mathrm{Cu}^{\mathrm{SUUL}}{ }_{20}$ & 79.2 & 21.1 & 10.9 & 8.19 & 1.46 & 54.1 & 3.86 & 12.7 \\
\hline $\mathrm{Cu}-\mathrm{NP}_{20}$ & 78.3 & 20.3 & 10.8 & 8.06 & 1.36 & 53.5 & 4.65 & 13.0 \\
\hline $\mathrm{Cu}-S U L_{10}$ & 78.9 & 20.0 & 10.9 & 8.17 & 1.41 & 53.0 & 4.60 & 12.4 \\
\hline $\mathrm{Cu}-\mathrm{NP}_{10}$ & 79.2 & 20.6 & 10.7 & 7.65 & 1.53 & 54.5 & 4.40 & 13.0 \\
\hline $\mathrm{Cu}_{\mathrm{SUL}}$ & 78.8 & 21.5 & 10.7 & 8.05 & 1.41 & 54.4 & 4.12 & 13.1 \\
\hline $\mathrm{Cu}-\mathrm{NP}_{2}$ & 79.0 & 20.5 & 11.0 & 8.14 & 1.48 & 54.2 & 3.71 & 12.6 \\
\hline \multicolumn{9}{|l|}{ Cu source } \\
\hline Cu-SUL & 78.9 & 20.9 & 10.8 & 8.13 & 1.43 & 53.9 & 4.19 & 12.7 \\
\hline Cu-NP & 78.8 & 20.5 & 10.8 & 7.95 & 1.46 & 54.0 & 4.25 & 12.9 \\
\hline \multicolumn{9}{|l|}{ Cu inclusion level, mg/kg } \\
\hline 20 & 78.8 & 20.7 & 10.8 & 8.12 & 1.41 & 53.7 & 4.28 & 12.9 \\
\hline 10 & 79.1 & 20.3 & 10.8 & 7.91 & 1.47 & 53.8 & 4.49 & 12.7 \\
\hline 2 & 78.9 & 21.0 & 10.9 & 8.09 & 1.45 & 54.3 & 3.91 & 12.9 \\
\hline SEM & 0.194 & 0.181 & 0.074 & 0.091 & 0.054 & 0.329 & 0.181 & 0.145 \\
\hline \multicolumn{9}{|l|}{$P$-values } \\
\hline Cu source (S) & 0.807 & 0.271 & 0.946 & 0.325 & 0.794 & 0.779 & 0.870 & 0.574 \\
\hline Cu inclusion level (IL) & 0.852 & 0.265 & 0.974 & 0.598 & 0.905 & 0.772 & 0.426 & 0.897 \\
\hline $\mathrm{IL} \times \mathrm{S}$ interaction & 0.388 & 0.130 & 0.382 & 0.406 & 0.717 & 0.433 & 0.371 & 0.292 \\
\hline
\end{tabular}

${ }_{1}^{1}$ diets supplemented with 20,10 and $2 \mathrm{mg}$ of copper sulphate $\left(\mathrm{Cu}-\mathrm{SUL}_{20}, \mathrm{Cu}-\mathrm{SUL}_{10}, \mathrm{Cu}-\mathrm{SUL}_{2}\right)$ or copper nanoparticles $\left(\mathrm{Cu}-\mathrm{NP}_{20}, \mathrm{Cu}-\mathrm{NP}_{10}\right.$, $\mathrm{Cu}-\mathrm{NP}_{2}$ ); SEM - standard error of the mean (standard deviation for all birds divided by the square root of the number of birds, $n=36$ )

Table 7. Redox status of fresh and frozen breast meat

\begin{tabular}{|c|c|c|c|c|c|c|c|c|}
\hline \multirow[b]{2}{*}{ Indices } & \multicolumn{4}{|c|}{ Fresh meat } & \multicolumn{4}{|c|}{ Frozen meat } \\
\hline & $\begin{array}{l}\mathrm{SOD} \\
\mathrm{U} / \mathrm{g}\end{array}$ & $\begin{array}{l}\text { CAT, } \\
\mathrm{U} / \mathrm{g}\end{array}$ & $\begin{array}{l}\text { GSH+GS } \\
\mu \mathrm{mol} / \mathrm{kg}\end{array}$ & $\begin{array}{l}\text { MDA, } \\
\mu \mathrm{mol} / \mathrm{kg}\end{array}$ & $\begin{array}{l}\mathrm{SOD} \\
\mathrm{U} / \mathrm{g}\end{array}$ & $\begin{array}{l}\text { CAT, } \\
\text { U/g }\end{array}$ & $\begin{array}{l}\text { GSH+GSSG, } \\
\mu \mathrm{mol} / \mathrm{kg}\end{array}$ & $\begin{array}{l}\mathrm{MDA}, \\
\mu \mathrm{mol} / \mathrm{kg}\end{array}$ \\
\hline \multicolumn{9}{|l|}{ Group $^{1}$} \\
\hline Cu-SUL 20 & 2.74 & 3.48 & 1.00 & $2.63^{\mathrm{a}}$ & 4.53 & 10.9 & 0.92 & 2.98 \\
\hline Cu-NP 20 & 3.70 & 4.11 & 0.96 & $1.92^{b}$ & 4.56 & 11.6 & 0.83 & 2.84 \\
\hline Cu-SUL $_{10}$ & 3.28 & 3.99 & 1.09 & $1.87^{\mathrm{b}}$ & 3.78 & 12.5 & 0.86 & 2.45 \\
\hline $\mathrm{Cu}-\mathrm{NP}_{10}$ & 3.56 & 5.82 & 1.02 & $1.86^{b}$ & 4.40 & 12.2 & 0.92 & 2.79 \\
\hline Cu-SUL & 3.27 & 4.21 & 1.15 & $2.01^{b}$ & 4.70 & 11.2 & 1.00 & 1.93 \\
\hline $\mathrm{Cu}^{-N P_{2}}$ & 3.55 & 5.30 & 1.10 & $2.62^{\mathrm{a}}$ & 5.17 & 12.0 & 0.98 & 2.70 \\
\hline \multicolumn{9}{|l|}{ Cu source } \\
\hline Cu-SUL & $3.10^{b}$ & $4.21^{b}$ & $1.08^{\mathrm{a}}$ & 2.17 & 4.34 & 11.5 & 0.93 & 2.45 \\
\hline Cu-NP & $3.61^{\mathrm{a}}$ & $5.30^{\mathrm{a}}$ & $1.03^{b}$ & 2.13 & 4.71 & 11.9 & 0.91 & 2.78 \\
\hline \multicolumn{9}{|l|}{ Cu inclusion level, mg/kg } \\
\hline 20 & 3.22 & $3.80^{\mathrm{b}}$ & $0.98^{c}$ & $2.28^{a}$ & $4.55^{\mathrm{ab}}$ & $11.3^{b}$ & $0.88^{b}$ & $2.91^{a}$ \\
\hline 10 & 3.42 & $4.91^{\mathrm{a}}$ & $1.06^{b}$ & $1.86^{b}$ & $4.09^{b}$ & $12.3^{\mathrm{a}}$ & $0.89^{b}$ & $2.62^{\mathrm{ab}}$ \\
\hline 2 & 3.41 & $4.75^{\mathrm{a}}$ & $1.13^{\mathrm{a}}$ & $2.32^{\mathrm{a}}$ & $4.93^{\mathrm{a}}$ & $11.6^{\mathrm{ab}}$ & $0.99^{a}$ & $2.31^{b}$ \\
\hline SEM & 0.118 & 0.170 & 0.014 & 0.071 & 0.123 & 0.169 & 0.017 & 0.097 \\
\hline \multicolumn{9}{|l|}{$P$-values } \\
\hline Cu source (S) & 0.033 & $<0.001$ & 0.025 & 0.722 & 0.107 & 0.200 & 0.582 & 0.069 \\
\hline Cu inclusion level (IL) & 0.732 & 0.002 & $<0.001$ & 0.002 & 0.016 & 0.024 & 0.013 & 0.027 \\
\hline $\mathrm{IL} \times \mathrm{S}$ interaction & 0.391 & 0.177 & 0.802 & $<0.001$ & 0.554 & 0.302 & 0.216 & 0.115 \\
\hline
\end{tabular}

${ }^{1}$ diets supplemented with 20,10 and $2 \mathrm{mg}$ of copper sulphate (Cu-SUL $\left.\left.{ }_{20}, \mathrm{Cu}-\mathrm{SUL}{ }_{10}, \mathrm{Cu}-\mathrm{SUL}\right)_{2}\right)$ or copper nanoparticles $\left(\mathrm{Cu}-\mathrm{NP}_{20}, \mathrm{Cu}_{-} \mathrm{NP}_{10}\right.$, Cu-NP $P_{2}$ ); SEM - standard error of the mean (standard deviation for all birds divided by the square root of the number of birds, $n=36$ ); $a, b$ - two-way ANOVA was applied, means within the same column with different superscript letters are significantly different across groups $(P \leq 0.05)$ in Tukey's test (calculated only if the IL $\times$ S interaction was significant) 
percentage, relative weights of major muscle groups (breast, thigh, drumstick) and breast meat colour were similar in all experimental subgroups.

The experimental factors differentiated most redox indicators in fresh breast meat and induced less pronounced differences in frozen breast meat (Table 7). The substitution of $\mathrm{Cu}-\mathrm{SUL}$ with $\mathrm{Cu}-$ NP in turkey diets increased SOD and CAT activities, and decreased the total glutathione content of fresh meat $(P<0.001, P=0.033$ and $P=0.025$, respectively). An analysis of MDA content revealed a significant interaction between the source and inclusion level of $\mathrm{Cu}$, where the highest and lowest dose of $\mathrm{Cu}-\mathrm{NP}$ led to a decrease and an increase in MDA levels, respectively. A decrease in the dietary $\mathrm{Cu}$ dose from $20 \mathrm{mg} / \mathrm{kg}$ to 10 and $2 \mathrm{mg} / \mathrm{kg}$ did not affect SOD activity, but increased CAT activity $(P=0.002)$ in fresh breast meat. In fresh breast meat, total glutathione content increased $(P<0.001)$ subject to the applied dose of supplemental $\mathrm{Cu}$, whereas MDA levels decreased in the group receiving a moderate dose of $\mathrm{Cu}$ and were not affected by the source of supplemental $\mathrm{Cu}$. The analysed redox parameters did not differ in frozen meat. The frozen meat of turkeys whose diets were supplemented with the lowest $\mathrm{Cu}$ dose was characterised by higher total glutathione content $(P<0.001)$ relative to the remaining groups) and significantly lower MDA levels $(P<0.027)$ relative to the group receiving the highest dose of supplemental $\mathrm{Cu}$.

\section{Discussion}

In the present experiment, supplemental $\mathrm{Cu}$ doses of 2, 10 and $20 \mathrm{mg} / \mathrm{kg}$ increased the total $\mathrm{Cu}$ content of turkey diets to approximately 14, 21 and $30 \mathrm{mg} / \mathrm{kg}$, respectively. The above values were lower, similar and higher, respectively, than the inclusion rate of $25 \mathrm{mg} / \mathrm{kg}$ recommended for poultry diets in the EU (EFSA FEEDAP Panel, 2016). The difference between the total $\mathrm{Cu}$ content in diets and supplemental $\mathrm{Cu}$ doses indicates that the major feed ingredients supplied approximately $11 \mathrm{mg} / \mathrm{kg} \mathrm{Cu}$ in total.

In the current study, dietary supplementation with $\mathrm{Cu}-\mathrm{NP}$ increased $\mathrm{Cu}$ concentration in the blood plasma, but only in turkeys whose diets were supplemented with the highest $\mathrm{Cu}$ dose. At the same time, plasma $\mathrm{Zn}$ levels decreased in response to higher plasma $\mathrm{Cu}$ levels and increased in response to lower plasma $\mathrm{Cu}$ levels (interaction between $\mathrm{Cu}$ source and $\mathrm{Cu}$ level). The $\mathrm{Cu}$ dose had no effect on $\mathrm{Ca}$ and $\mathrm{Mg}$ levels in the blood, and the only change observed was in blood P levels which increased in response to lower dietary $\mathrm{Cu}$ supplementation. The correlation between $\mathrm{Cu}-\mathrm{NP}$ supplementation and intestinal absorption of $\mathrm{Zn}$ as also confirmed in the study on chickens (Ognik et al., 2016). The cited authors observed that intestinal absorption of $\mathrm{Zn}$ was improved in line with a decrease in the dietary dose of $\mathrm{Cu}$ nanoparticles. Copper and $\mathrm{Zn}$ concentrations in cells are regulated by metallothioneins (MTs), which are low-molecular-weight proteins abundant in cysteine residues. A single MT molecule is capable of binding seven divalent $\mathrm{Zn}$ ions and up to 12 monovalent $\mathrm{Cu}$ ions. Due to similarities in the coordination chemistry of $\mathrm{Zn}$ and $\mathrm{Cu}, \mathrm{Cu}$ is able to compete for $\mathrm{Zn}$ binding sites and push $\mathrm{Zn}$ away from the molecule (Gaetke and Chow, 2003).

The results of previous studies investigating the effect of dietary $\mathrm{Cu}$ levels on microelement and macroelement concentrations in the blood are inconclusive. Chickens fed diets supplemented with $75 \mathrm{mg} \mathrm{Cu}-\mathrm{SUL} / \mathrm{kg}$ were characterised by increased plasma concentrations of $\mathrm{Cu}$ and $\mathrm{Zn}$ at 42 days of age, relative to control group birds whose diets were not supplemented with $\mathrm{Cu}$ (Samanta et al., 2011). In another experiment, dietary supplementation with $\mathrm{Cu}$ at $50 \mathrm{mg} / \mathrm{kg}$ increased $\mathrm{Cu}$ concentration, decreased $\mathrm{Zn}$ concentration and had no significant effect on $\mathrm{Fe}$ concentration in the blood plasma of 8-week-old chickens. In 18-week-old birds, dietary $\mathrm{Cu}$ had no influence on the plasma concentrations of $\mathrm{Zn}, \mathrm{Fe}$ or Mn (Adegbenjo et al., 2014). Bao et al. (2007) reported similar concentrations of trace minerals in the blood plasma of chickens fed diets supplemented with 4 and $40 \mathrm{mg} \mathrm{Cu} / \mathrm{kg}$ (Bioplex-Cu). In our experiment, supplemental $\mathrm{Cu}$ doses of 2,10 and $20 \mathrm{mg} / \mathrm{kg}$ did not differentiate $\mathrm{Cu}, \mathrm{Zn}$ or $\mathrm{Mg}$ levels in the blood plasma.

Copper plays an important role in Fe metabolism, $\mathrm{Hb}$ synthesis and erythrocyte production (Tapiero et al., 2003; Mroczek-Sosnowska et al., 2013; Ognik et al., 2018) because ceruloplasmin transports around $95 \%$ of $\mathrm{Cu}$ in the blood stream and also participates in iron metabolism. Therefore, red blood cell indicators ( $\mathrm{RBC}, \mathrm{Hb}$ ) increased in the few experiments where chicken diets were supplemented with $\mathrm{Cu}$ nanoparticles (Mroczek-Sosnowska et al., 2013; Miroshnikov et al., 2015; Ognik et al., 2018). In the present experiment, the substitution of $\mathrm{Cu}-\mathrm{SUL}$ with $\mathrm{Cu}-\mathrm{NP}$ increased $\mathrm{Hb}$ values. In broiler chickens, plasma $\mathrm{Hb}$ levels increased when diets were supplemented with $75-150 \mathrm{mg} \mathrm{Cu} / \mathrm{kg}$ relative to the control group, whereas a decrease in plasma $\mathrm{Hb}$ levels was noted in response to the 
highest $\mathrm{Cu}$ dose of $250 \mathrm{mg} / \mathrm{kg}$ (Samanta et al., 2011). In an experiment performed on growing turkeys (Makarski et al., 2014), a dietary Cu-SUL dose of $50 \mathrm{mg} / \mathrm{kg}$ decreased $\mathrm{Hb}$ levels relative to the control group where the diet was not supplemented with $\mathrm{Cu}$. In the present study, diets supplemented with up to $30 \mathrm{mg} \mathrm{Cu} / \mathrm{kg}$ did not induce changes in $\mathrm{Hb}$ levels in growing turkeys. In a study by Ognik et al. (2018), haematological parameters were influenced by the inclusion levels of $\mathrm{Cu}$ nanoparticles in chicken diets. In treatments where $\mathrm{Cu}$ levels were below the values recommended by the National Research Council (NRC, 1994), the supplementation of chicken diets with $\mathrm{Cu}$ nanoparticles increased $\mathrm{Hb}$ and $\mathrm{Ht}$ levels as well as RBC counts. Haemoglobin, $\mathrm{Ht}$ and $\mathrm{RBC}$ values decreased in chickens when the total $\mathrm{Cu}$ content in diets increased by $13 \%$ or more in excess of the recommended levels (NRC, 1994) due to supplementation with $\mathrm{Cu}$ nanoparticles. Ghasemipoor and Zolghadri (2014) also reported a decrease in the $\mathrm{Hb}$ levels of chickens whose diets were supplemented with nano- $\mathrm{CuO}$ at $16 \mathrm{mg} / \mathrm{kg}$ BW for 35 days compared with birds fed control unsupplemented diets.

In our experiment on young turkeys, the dietary supplementation with $\mathrm{Cu}-\mathrm{NP}$, in particular at the inclusion level of $20 \mathrm{mg} / \mathrm{kg}$, decreased plasma glucose concentrations in turkeys (Kozłowski et al., 2018). Mroczek-Sosnowska et al. (2016) also observed a decrease in plasma glucose levels of chickens administered $\mathrm{Cu}-\mathrm{NP}$ in ovo during embryogenesis. However, in the present study, the above effects were not confirmed when $\mathrm{Cu}$-SUL was replaced with $\mathrm{Cu}-\mathrm{NP}$ and when the dose of supplemental $\mathrm{Cu}$ was decreased. No differences were found in most blood biochemical parameters of turkeys fed diets with graded $\mathrm{Cu}$ levels.

A model study revealed that $\mathrm{Cu}$ is able to downregulate lipid metabolism, especially cholesterol biosynthesis (Huster and Lutsenko, 2007). The above could partly explain the results of the present study where total plasma cholesterol concentration was the highest in turkeys fed diets with the lowest dose of supplemental $\mathrm{Cu}$. Similar findings were reported by Kaya et al. (2006) who observed symptoms of hypertriglyceridemia, hypercholesterolemia and anaemia in chickens fed diets deficient in $\mathrm{Cu}$ ( $3.5 \mathrm{mg} \mathrm{Cu} / \mathrm{kg}$ in feed ingredients) relative to birds fed diets containing $8 \mathrm{mg} \mathrm{Cu} / \mathrm{kg}$.

According to many studies, $\mathrm{Cu}$ can promote growth in chickens at dietary doses of 100 to $450 \mathrm{mg} / \mathrm{kg}$ (Pekel and Alp, 2011; Samanta et al., 2011). However, Sawosz et al. (2018) observed that the standard inclusion level of $\mathrm{Cu}(7.5 \mathrm{mg} / \mathrm{kg}$ of the diet) recommended by the NRC (1994) can significantly improve chicken performance if $25 \%$ of the $\mathrm{CuSO}_{4}$ dose is replaced with $\mathrm{Cu}$ nanoparticles. Mroczek-Sosnowska et al. (2016) confirmed the beneficial effect of $\mathrm{Cu}$ nanoparticles injected in ovo on the performance of growing chickens. However, in the present study, the replacement of $\mathrm{Cu}-\mathrm{SUL}$ with $\mathrm{Cu}-\mathrm{NP}$ and the decrease in the dose of supplemental $\mathrm{Cu}$ from $20 \mathrm{mg} / \mathrm{kg}$ to 10 and $2 \mathrm{mg} / \mathrm{kg}$ did not affect the growth performance or the carcass quality of turkeys. In one of the few experiments performed on growing turkeys, the supplementation of bird diets with $50 \mathrm{mg} \mathrm{Cu}-\mathrm{SUL} / \mathrm{kg}$ did not induce changes in growth performance (Makarski et al., 2014). In the present experiment, the growth performance of birds and slaughter yield were similar in the compared treatments, regardless of the dose of supplemental $\mathrm{Cu}$. The above could indicate that the $\mathrm{Cu}$ content of basic feed components (approx. $11 \mathrm{mg} / \mathrm{kg}$ ) fulfilled the nutrient requirements of turkeys. This observation is also supported by similar slaughter yield and carcass quality traits in the analysed turkeys.

It is generally accepted that the oxidative stability of meat can be improved with diet modification (Estévez, 2015). For this reason, poultry diets are supplemented with antioxidants, such as vitamin $\mathrm{E}$ and selenium, to protect meat against oxidative damage. Research has demonstrated that $\mathrm{Cu}$ may act as an antioxidant or a pro-oxidant in living birds, depending on the dose and other feeding parameters (Ajuwon and Idowu, 2010; Xu et al., 2012). High $\mathrm{Cu}$ concentrations decreased glutathione levels and 3-hydroxy-3-methyl-glutaryl-coenzyme A (HMG-CoA) reductase activity, and limited cholesterol synthesis (Bakalli et al., 1995). Broilers whose diets were supplemented with $250 \mathrm{mg}$ $\mathrm{Cu}-\mathrm{SUL} / \mathrm{kg}$ for 6 weeks were characterised by increased lipid peroxidation in the liver and plasma, lower CAT activity and lower levels of SOD and GSH (Ajuwon et al., 2011). However, the elimination of iron and $\mathrm{Cu}$ from the diet decreased oxidation values in cooked broiler leg meat in the thiobarbituric acid reactive substances assay (Ruiz et al., 2000). Bozkaya et al. (2001) observed intensified lipid peroxidation, an increase in MDA levels, a decrease in $\mathrm{Cu}$-dependent SOD activity, and an increase in CAT activity in chickens fed $\mathrm{Cu}$-deficient diets. In the current study, the replacement of $\mathrm{Cu}-\mathrm{SUL}$ with $\mathrm{Cu}-\mathrm{NP}$ increased SOD and CAT activity and decreased the total glutathione content of fresh breast meat. In frozen 
meat, the above parameters were similar regardless of the source of supplemental $\mathrm{Cu}$. In fresh meat, the optimal ratio of total glutathione (average values) to MDA (lowest value relative to the remaining treatments) was noted when turkey diets were supplemented with a moderate dose of $\mathrm{Cu}$. In frozen meat, glutathione content was the highest and MDA content was the lowest when turkey diets were supplemented with the lowest $\mathrm{Cu}$ dose. A decrease in the glutathione content of meat could indicate that $\mathrm{Cu}$ nanoparticles lowered the antioxidant potential of meat, which stimulated SOD and CAT activity. However, the observed effect was moderate and short-lived, it did not increase MDA levels and was not confirmed in frozen meat. A decrease in the dose of supplemental $\mathrm{Cu}$ from $20 \mathrm{mg} / \mathrm{kg}$ to 10 and $2 \mathrm{mg} / \mathrm{kg}$ did not compromise the antioxidant potential of meat. The optimal redox parameters were noted in the fresh meat of turkeys receiving a moderate $\mathrm{Cu}$ dose and in the frozen meat of turkeys receiving the lowest $\mathrm{Cu}$ dose.

\section{Conclusions}

It can be concluded that the replacement of $\mathrm{Cu}$ sulphate (Cu-SUL) with $\mathrm{Cu}$ nanoparticles (Cu-NP) and a decrease in the dose of supplemental $\mathrm{Cu}$ from 20 to $10 \mathrm{mg} / \mathrm{kg}$ or even $2 \mathrm{mg} / \mathrm{kg}$ of the diet did not affect the growth parameters or the carcass quality of turkeys.

The few effects exerted by the substitution of $\mathrm{Cu}-\mathrm{SUL}$ with $\mathrm{Cu}-\mathrm{NP}$ included an increase in haemoglobin levels and an improvement in the antioxidant status of fresh breast meat. However, fresh meat was characterised by optimal redox parameters when the dietary dose of $\mathrm{Cu}$ was decreased to $10 \mathrm{mg} / \mathrm{kg}$. The results of the present study cannot be generalised, but they significantly expand our knowledge about $\mathrm{Cu}-\mathrm{NP}$ as an efficient source of $\mathrm{Cu}$ for turkeys.

\section{Acknowledgments}

The study was carried out as part of the Biostrateg program entitled 'GUTFEED - innovative nutrition in sustainable poultry production', grant No. 267659/7/NCBR/2015.

\section{References}

Adegbenjo A.A., Idowu O.M.O., Oso A.O., Adeyemi O.A., Sobayo R.A., Akinloye O.A., Jegede A.V., Osho S.O., Williams G.A., 2014. Effects of dietary supplementation with copper sulphate and copper proteinate on plasma trace minerals, copper residues in meat tissues, organs, excreta and tibia bone of cockerels. Slovak J. Anim. Sci. 47, 164-171
Ajuwon O.R., Idowu O.M.O., 2010. Vitamin C attenuated copper-induced oxidative damage in broiler chickens. Afr. J. Biotechnol. 9, 7525-7530, https://doi.org/10.5897/AJB10.776

Ajuwon O.R., Idowu O.M.O., Afolabi S.A., Kehinde B.O., Oguntola O.O., Olatunbosun K.O., 2011. The effects of dietary copper supplementation on oxidative and antioxidant systems in broiler chickens. Arch. Zootec. 60, 275-282, https://doi. org/10.4321/S0004-05922011000200012

Andersen O., 2004. Chemical and biological considerations in the treatment of metal intoxications by chelating agents. Mini Rev. Med. Chem. 4, 11-21, https://doi.org/10.2174/1389557043487583

Bakalli R.I., Pesti G.M., Ragland W.L., Konjufca V., 1995. Dietary copper in excess of nutritional requirement reduces plasma and breast muscle cholesterol of chickens. Poult. Sci. 74, 360365, https://doi.org/10.3382/ps.0740360

Bao Y.M., Choct M., lji P.A., Bruerton K., 2007. Effect of organically complexed copper, iron, manganese, and zinc on broiler performance, mineral excretion, and accumulation in tissues. J. Appl. Poult. Res. 16, 448-455, https://doi.org/10.1093/ japr/16.3.448

Bozkaya L.A., Öztürk-Ürek R., Aydemir T., Tarhan L., 2001. Effects of $\mathrm{Se}, \mathrm{Cu}$ and $\mathrm{Se}+$ vitamin $\mathrm{E}$ deficiency on the activities of CuZnSOD, GSH-Px, CAT and LPO levels in chicken erythrocytes. Cell Biochem. Funct. 19, 153-157, https://doi. org/10.1002/cbf.906

EFSA FEEDAP Panel (EFSA Panel on Additives and Products or Substances used in Animal Feed), 2016. Scientific opinion on the revision of the currently authorised maximum copper content in complete feed. EFSA J. 14, 4563, https://doi.org/10.2903/j. efsa.2016.4563

Estévez M., 2015. Oxidative damage to poultry: from farm to fork. Poult. Sci. 94, 1368-1378, https://doi.org/10.3382/ps/pev094

European Commission, 2003. Opinion of the Scientific Committee for Animal Nutrition on the use of copper in feedingstuffs. https:// ec.europa.eu/food/sites/food/files/safety/docs/animal-feed_ additives_rules_scan-old_report_out115.pdf

Gaetke L.M., Chow C.K., 2003. Copper toxicity, oxidative stress, and antioxidant nutrients. Toxicology 189, 147-163, https://doi. org/10.1016/S0300-483X(03)00159-8

Ghasemipoor M., Zolghadri S., 2014. The effect of copper oxide nanoparticles as feed additive on some the blood proteins of broiler chickens. Mol. Cell Biol. Res. Commun. 3, Suppl. 1,144

Gonzales-Eguia A., Fu C.-M., Lu F.-Y., Lien T.-F., 2009. Effects of nanocopper on copper availability and nutrients digestibility, growth performance and serum traits of piglets. Livest. Sci. 126, 122-129, https://doi.org/10.1016/j.livsci.2009.06.009

Huster D., Lutsenko S., 2007. Wilson disease: not just a copper disorder. Analysis of a Wilson disease model demonstrates the link between copper and lipid metabolism. Mol. Biosyst. 3, 816-824, https://doi.org/10.1039/B711118P

Hybrid Turkeys, 2016. Nutrient Guidelines. https://resources. hybridturkeys.com/nutrition/commercial-guidelines/ (accessed 2.01.2019)

Jankowski J., Kozłowski K., Ognik K., Zduńczyk Z., Otowski K., Sawosz E., Juśkiewicz J., 2019. Redox and immunological status of turkeys fed diets with different levels and sources of copper. Ann. Anim. Sci. 19, 215-227, https://doi.org/10.2478/ aoas-2018-0054

Jegede A.V., Oduguwa O.O., Bamgbose A.M., Fanimo A.O., Nollet L., 2011. Growth response, blood characteristics and copper accumulation in organs of broilers fed on diets supplemented with organic and inorganic dietary copper sources. Br. Poult. Sci. 52, 133-139, https://doi.org/10.1080/00071668.2010.54 4714 
Kaya A., Altıner A., Özpınar A., 2006. Effect of copper deficiency on blood lipid profile and haematological parameters in broilers. J. Vet. Med. Ser. A 53, 399-404, https://doi.org/10.1111/ j.1439-0442.2006.00835.x

Kozłowski K., Jankowski J., Otowski K., Zduńczyk Z., Ognik K., 2018. Metabolic parameters in young turkeys fed diets with different inclusion levels of copper nanoparticles. Pol. J. Vet. Sci. 21, 245-253, https://doi.org/10.24425/119043

Leeson S., Caston L., 2008. Using minimal supplements of trace minerals as a method of reducing trace mineral content of poultry manure. Anim. Feed Sci. Technol. 142, 339-347, https://doi.org/10.1016/j.anifeedsci.2007.08.004

Makarski B., Gortat M., Lechowski J., Żukiewicz-Sobczak W., Sobczak P., Zawiślak K., 2014. Impact of copper (Cu) at the dose of $50 \mathrm{mg}$ on haematological and biochemical blood parameters in turkeys, and level of $\mathrm{Cu}$ accumulation in the selected tissues as a source of information on product safety for consumers. Ann. Agric. Environ. Med. 21, 567-570, https://doi.org/10.5604/12321966.1120603

Mikulski D., Jankowski J., Zduńczyk Z., Wróblewska M., Mikulska M., 2009. Copper balance, bone mineralization and the growth performance of turkeys fed diet with two types of $\mathrm{Cu}$ supplements. J. Anim. Feed Sci. 18, 677-688, https://doi. org/10.22358/jafs/66441/2009

Miroshnikov S.A., Yausheva E.V., Sizova E.A., Miroshnikova E.P., Levahin V.L., 2015. Comparative assessment of effect of copper nano- and microparticles in chicken. Orient. J. Chem. 31, 2327-2336, https://doi.org/10.13005/ojc/310461

Mroczek-Sosnowska N., Batorska M., Łukasiewicz M., Wnuk A., Sawosz E., Jaworski S., Niemiec J., 2013. Effect of nanoparticles of copper and copper sulfate administered in ovo on hematological and biochemical blood markers of broiler chickens. Ann. Warsaw Univ. Life Sci. SGGW Anim. Sci. 52, 141-149

Mroczek-Sosnowska N., Łukasiewicz M., Wnuk A., Sawosz E., Niemiec J., Skot A., Jaworski S., Chwalibog A., 2016. In ovo administration of copper nanoparticles and copper sulfate positively influences chicken performance. J. Sci. Food Agric. 96, 3058-3062, https://doi.org/10.1002/jsfa.7477

NRC (National Research Council), 1994. Nutrient Requirements of Poultry. $9^{\text {th }}$ Revised Edition. The National Academies Press. Washington, DC (USA), https://doi.org/10.17226/2114

Ognik K., Wertelecki T., 2012. Effect of different vitamin E sources and levels on selected oxidative status indices in blood and tissues as well as on rearing performance of slaughter turkey hens. J. Appl. Poult. Res. 2, 259-271, https://doi.org/10.3382/ japr.2011-00366
Ognik K., Steppniowska A., Cholewińska E., Kozłowski K., 2016. The effect of administration of copper nanoparticles to chickens in drinking water on estimated intestinal absorption of iron, zinc, and calcium. Poult. Sci. 95, 2045-2051, https://doi. org/10.3382/ps/pew200

OgnikK., Sembratowicz I., Cholewińska E., Jankowski J., Kozłowski K., Juśkiewicz J., Zduńczyk Z., 2018. The effect of administration of copper nanoparticles to chickens in their drinking water on the immune and antioxidant status of the blood. Anim. Sci. J. 89, 579-588, https://doi.org/10.1111/asj.12956

Pekel A.Y., Alp M., 2011. Effects of different dietary copper sources on laying hen performance and egg yolk cholesterol. J. Appl. Poult. Res. 20, 506-513, https://doi.org/10.3382/japr.201000313

Pineda L., Sawosz E., Vadalasetty K.P., Chwalibog A., 2013. Effect of copper nanoparticles on metabolic rate and development of chicken embryos. Anim. Feed Sci. Technol. 186, 125-129, https://doi.org/10.1016/j.anifeedsci.2013.08.012

Ruiz J.A., Perez-Vendrell A.M., Esteve-Garcia E., 2000. Effect of dietary iron and copper on performance and oxidative stability in broiler leg meat. Br. Poult. Sci. 41, 163-167, https://doi. org/10.1080/713654910

Samanta B., Ghosh P.R., Biswas A., Das S.K., 2011. The effects of copper supplementation on the performance and hematological parameters of broiler chickens. AsianAustralas. J. Anim. Sci. 24, 1001-1006, https://doi. org/10.5713/ajas.2011.10394

Sawosz E., Łukasiewicz M., Łozicki A., Sosnowska M., Jaworski S., Niemiec J., Scott A., Jankowski J., Józefiak D., Chwalibog A., 2018. Effect of copper nanoparticles on the mineral content of tissues and droppings, and growth of chickens. Arch. Anim. Nutr. 72, 396-406, https://doi.org/10.1080/174503 9X.2018.1505146

Skřivan M., Skřivanová V., Marounek M., 2006. Effect of various copper supplements to feed of laying hens on Cu content in eggs, liver, excreta, soil, and herbage. Arch. Environ. Contam. Toxicol. 50, 280-283, https://doi.org/10.1007/ s00244-005-1028-1

Tapiero H., Townsend D.M., Tew K.D., 2003. Trace elements in human physiology and pathology. Copper. Biomed. Pharmacother. 57, 386-398, https://doi.org/10.1016/S07533322(03)00012-X

Xu P., Xu J., Liu S., Ren G., Yang Z., 2012. In vitro toxicity of nanosized copper particles in PC12 cells induced by oxidative stress. J. Nanopart. Res. 14, 906, https://doi.org/10.1007/s11051. 012-0906-5 